\title{
悬 \\ Interesse comportamental de machos suínos por diferentes tipos de enriquecimentos ambientais
}

[Behavioral interest of male swine for different types of environmental enrichment]

"Artigo Científico/Scientific Article"

\author{
Gisele Dela Ricci ${ }^{1}$, Elder Tonon ${ }^{1}$, Cristiane Gonçalves Titto ${ }^{1}$, Paula Caroline Godoy ${ }^{2}$ \\ Evaldo Lencioni Titto ${ }^{3}$
}

\begin{abstract}
${ }^{1}$ Departamento de Zootecnia, Faculdade de Zootecnia e Engenharia de Alimentos, Universidade de São Paulo, Pirassununga-SP, Brasil.

${ }^{2}$ Departamento de Engenharia de Biossistemas, Faculdade de Zootecnia e Engenharia de Alimentos, Universidade de São Paulo, Pirassununga-SP, Brasil.

${ }^{3}$ Departamento de Ciências Básicas, Faculdade de Zootecnia e Engenharia de Alimentos, Universidade de São Paulo, Pirassununga-SP, Brasil.

*Autor para correspondência/Corresponding author: E-mail: giseledelaricci@usp.br
\end{abstract}

\begin{abstract}
Resumo
O objetivo deste estudo foi avaliar o comportamento de machos suínos a partir da inserção de correntes e pneus como enriquecimento ambiental em confinamento. O estudo foi realizado na Universidade de São Paulo, em Pirassununga - SP, Brasil. Foram utilizados seis machos híbridos, de 12 a 30 meses. Foram inseridos correntes e pneus, individualmente. O comportamento e o tempo de interesse pelo enriquecimento foi obtido observando o momento em que o animal procurava pelo objeto inserido, com observações diretas, sem intervalos, de manhã e à tarde. Foram aferidas as temperaturas superficiais do corpo dos animais e ambientais, com obtenção da frequência respiratória no início e no final das observações. Foram realizadas colheitas de cortisol salivar, por meio de corda de algodão individualmente. Foi realizada análise de tipo de enriquecimento e idade por análise de qui-quadrado, correlação de Pearson entre o comportamento e temperatura de superfície corporal e teste-t para frequência respiratória e temperatura do ambiente. A idade dos cachaços não teve influência no comportamento de interesse $(\mathrm{P}>0,05)$. No total, 79,86\% dos machos não apresentaram interesse pela corrente, no entanto, $61,11 \%$ dos machos apresentaram interesse pelo pneu. No ambiente controle, $91,67 \%$ dos animais permaneceram em ócio. A frequência respiratória encontrada foi maior a tarde $(\mathrm{P}<0,05)$. As dosagens séricas de cortisol, foram de 0,5 a $3,1 \mathrm{ng} / \mathrm{mL}, 0,5$ a $0,9 \mathrm{ng} / \mathrm{mL}, 0,5$ a $1,2 \mathrm{ng} / \mathrm{mL}$ e de 0,9 a $1,2 \mathrm{ng} / \mathrm{mL}$. Conclui-se que o enriquecimento ambiental nas instalações de suínos machos constitui-se como importante ferramenta, sendo o pneu o melhor método de entretenimento quando comparado ao uso de correntes.
\end{abstract}

Palavras-chave: ambiente; bem-estar animal; correntes; infravermelho; pneus.

\begin{abstract}
The objective of this study was to evaluate the behavior of male swine after the insertion of chains and tires as environmental enrichment in confinement. The study was carried out at the University of São Paulo, in Pirassununga - SP, Brazil. Six hybrid males, 12 to 30 months of age, were used. Chains and tires were inserted individually. The behavior and the time of interest for the enrichment was obtained observing the moment in which the animal looked for the inserted object, with direct observations, without intervals, morning and afternoon. The ambient and body temperatures were measured, with respiratory rate obtained at the beginning and at the end of the observations. Salivary cortisol was obtained by means of an individual cotton rope. Enrichment type and age analysis were performed by chi-square analysis, Pearson's correlation between body surface temperature and behavior, and t-test for respiratory rate and ambient temperature. The age of the male swine had no influence on the behavior of interest. In total, $79.86 \%$ of the males showed no interest in the chains, however, $61.11 \%$ of the males showed interest in the tire. In the control environment, $91.67 \%$ of the animals remained idle. The respiratory rate found was higher in the afternoon $(\mathrm{P}<0.05)$. Serum cortisol dosages
\end{abstract}


were 0.5 to $3.1 \mathrm{ng} / \mathrm{mL}, 0.5$ to $0.9 \mathrm{ng} / \mathrm{mL}, 0.5$ to $1.2 \mathrm{ng} / \mathrm{mL}$ and 0.9 to $1.2 \mathrm{ng} / \mathrm{mL}$. It is concluded that the environmental enrichment in the facilities of male pigs constitutes an important tool, being the tire the best method of entertainment when compared to the use of chains.

Keywords: environment; animal welfare; chains; infrared; tires.

\section{Introdução}

A carência de recursos ambientais adequados e o manejo inadequado podem determinar alterações no comportamento, induzindo a prejuízos no bem-estar e na produção animal (Sarubbi, 2010).

Quando alojados, em um determinado sistema de criação, é necessário que fatores como os níveis de crescimento e de reprodução, funcionamento ideal fisiológico e comportamental sejam avaliados, de acordo com a forma como os animais se comportam ao se relacionarem com o ambiente e a influência do estresse causado por ele, permitindo avaliações mais adequadas do bemestar animal (Manteca et al., 2013).

Estímulos ambientais que sobrecarreguem a fisiologia de controle, podem diminuir a adaptação, resultando em estresse, modificando a fisiológica, com aumento da frequência respiratória, diminuindo o bem-estar dos animais (Fraser e Broom, 1990). A frequência respiratória pode variar extrinsecamente em razão de fatores relacionados ao ambiente, como temperatura e umidade do ar, radiação solar, velocidade do vento, período do dia, sombreamento, estação do ano e densidade de alojamento dos suínos (Pereira, 2017).

O estresse crônico pode gerar concentrações elevadas de cortisol, reduzindo a aptidão individual o que acarreta imunossupressão e decréscimo da imunidade do animal, trazendo quedas na atividade reprodutiva e apresentações de comportamentos estereotipados (Möstl e Palme, 2002). Sua quantificação no soro sanguíneo é realizada para verificar o nível de estresse do animal durante sistema de criação em que foi submetido (Santana et al., 2009). Entretanto, a colheita de sangue é uma atividade que gera estresse, podendo afetar o desempenho animal (BRASIL, 2006), tendo como alternativa a colheita de saliva para mensuração do cortisol bem vista pela sua facilidade de colheita (Baptista et al., 2011).

A garantia da manutenção da temperatura de conforto ambiental apresenta importância fundamental para suínos machos em fase de reprodução sobre confinamento. A temperatura de conforto é definida como aquela que não é necessária qualquer atividade metabólica para manter o organismo animal aquecido ou resfriado (Oliveira et al., 2003). O calor e umidade elevada geram estresse crônico, sobretudo se seguidos de amplas flutuações de temperatura, resultando em redução na ingestão de alimento e interferência na espermatogênese dos machos (Kunavongkrit et al., 2005).

O estresse tem sido considerado como principal método de avaliação do bem-estar animal (Ferreira, 2004), buscando-se identificar fatores que possam reduzir a qualidade de vida dentro do confinamento. $\mathrm{O}$ bem-estar animal envolve percepções físicas e psicológicas em uma amplitude de características, podendo ser classificado em uma escala que varia entre o muito bom e o muito ruim (Broom e Molento, 2004).

Neste contexto, o enriquecimento ambiental surge como uma alternativa para melhorar a qualidade do ambiente de animais confinados, a partir de estímulos diversos, que sejam capazes de oferecer bem-estar psíquico e fisiológico, com a estimulação de comportamentos típicos dos animais (Vasconcelos et al., 2015). O enriquecimento ambiental melhora o bem-estar, permitindo a apresentação de comportamentos típicos da espécie, atuando no desenvolvimento físico e psicológico dos animais (Foppa et al., 2014), e atualmente é classificado de acordo com a atividade que exerce dentro do confinamento, as quais são classificadas como social, ocupacional, físico, sensorial e nutricional (Bloomsmith et al., 1991).

Para Maia et al. (2013), o ambiente pode ser enriquecido, ofertando artefatos aos animais que permitam a eles diversificar o seu comportamento. Suínos criados em ambientes enriquecidos apresentam comportamentos que indicam melhor bem-estar (Vasconcelos et al., 2015). É importante que os brinquedos oferecidos como enriquecimento sejam de fácil aplicação e que custem pouco para os produtores, sendo, correntes, cordas e garrafas PET (Scott et al., 2009; Foppa et al., 2014). O termo enriquecimento implica em melhorias, sejam elas físicas, sociais, alimentares entre outras, aplicadas como forma de alterar de modo favorável ao ambiente (Newberry, 1995).

A disponibilização de enriquecimentos ambientais atenua a apresentação de interações 
negativas, como a caudofagia, comportamentos estereotipados e agressividade entre os suínos (Guy et al., 2002; Rodarte et al., 2004). A inserção de bolas ou pequenos objetos de borracha reduzem o ócio, aumentam interações positivas e comportamentos lúdicos de suínos (Guy et al., 2002). No entanto, é necessário que a inserção do objeto seja planejada, para que este atenda à expectativa da espécie e se evite a frustação dos animais (Mench, 1998; Van de Weerd et al., 2006), causado perda do interesse pelo objeto (Campos et al., 2010). Com relação a machos reprodutores ainda, não existem muitas informações sobre sua utilização de enriquecimentos ambientais (Van de Weerd e Day, 2009). Desde 2003, a legislação europeia prevê a utilização de objetos para enriquecimento ambiental que estimulem e proporcionem o comportamento investigatório de suínos a partir das diretrizes 2001/88/EC e 2001/93/EC (Scott et al., 2009; Guy et al., 2013).

De acordo com a importância do enriquecimento ambiental para a categoria de suínos machos (reprodutores) confinados, o objetivo deste estudo foi avaliar o comportamento de interesse de cachaços, de diferentes idades, por correntes e pneus utilizados como enriquecimento ambiental em instalações em confinamento.

\section{Material e Métodos}

O experimento foi realizado no Setor de Suinocultura da Prefeitura da Universidade de São Paulo, Campus Fernando Costa, em Pirassununga, São Paulo. Foram utilizados seis machos híbridos, sendo quatro de 12 e dois de 30 meses de idade, com peso médio de $\pm 350 \mathrm{~kg}$ alojados em baias individuais de $24 \mathrm{~m}^{2}$, constituídas por piso de cimento na área coberta, com telhado de madeira, e piso de areia na área descoberta, com comedouros de cimento e bebedouro tipo chupeta, em baias iguais para ambos os enriquecimentos inseridos. Todos os machos passaram pelo tratamento controle e pelos enriquecimentos.

Os enriquecimentos oferecidos foram correntes e pneus. Realizou-se cinco dias de observação, nos quais os objetos foram oferecidos de forma alternada, um por dia, individualmente. Todos os animais receberam os dois tipos de enriquecimento oferecidos. Nos dias 1 e 3 foram inseridas as correntes, penduradas por uma corda, na altura da cabeça dos cachaços, aproximadamente $40 \mathrm{~cm}$. As correntes possuíam $30 \mathrm{~cm}$ de comprimento, constituídas de ferro. Os pneus utilizados foram os de aro 14, de veículos de passeio, inseridos nos dias 2, 4 e 5 de observação. Após a higienização, os pneus eram inseridos no chão da baia. O intuito da utilização dos pneus foi proporcionar a oportunidade dos machos arrastar no interior da baia.

O comportamento de interesse dos machos pelo enriquecimento foi obtido buscando observar o momento em que o animal apresentava interesse pelo objeto inserido ou não, sendo pontuado 0 para a não procura e 1 pela procura (Figuras 1 e 2). A frequência de utilização do enriquecimento foi realizada durante duas horas, a cada 10 minutos, no período da manhã e da tarde, durante os cinco dias, pelo método direto e focal (Tabela 1).

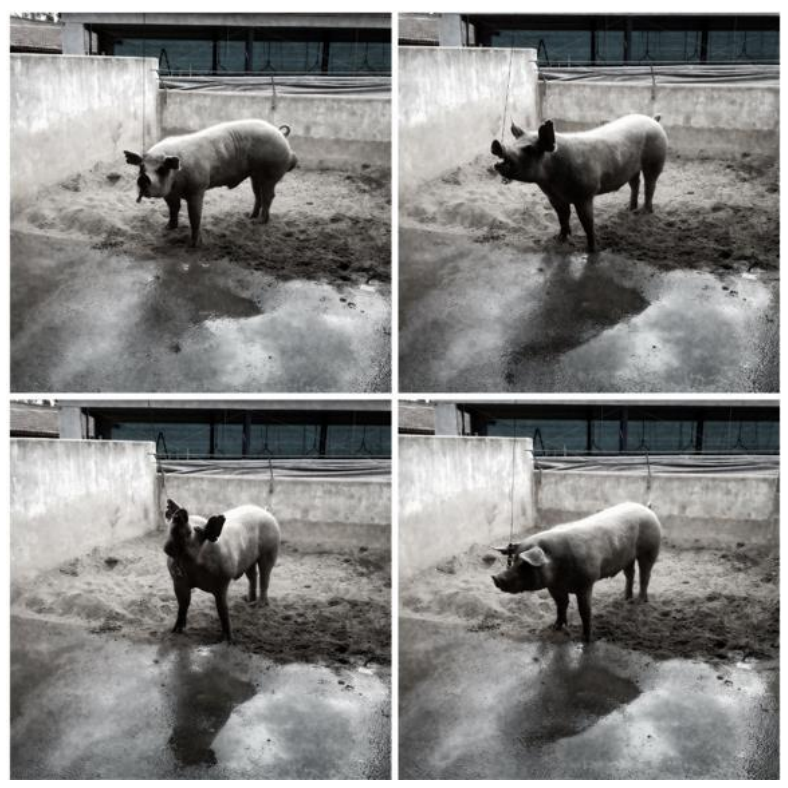

Figura 1. Sequência de interesse e desinteresse de cachaços em relação a corrente utilizado como enriquecimento ambiental.

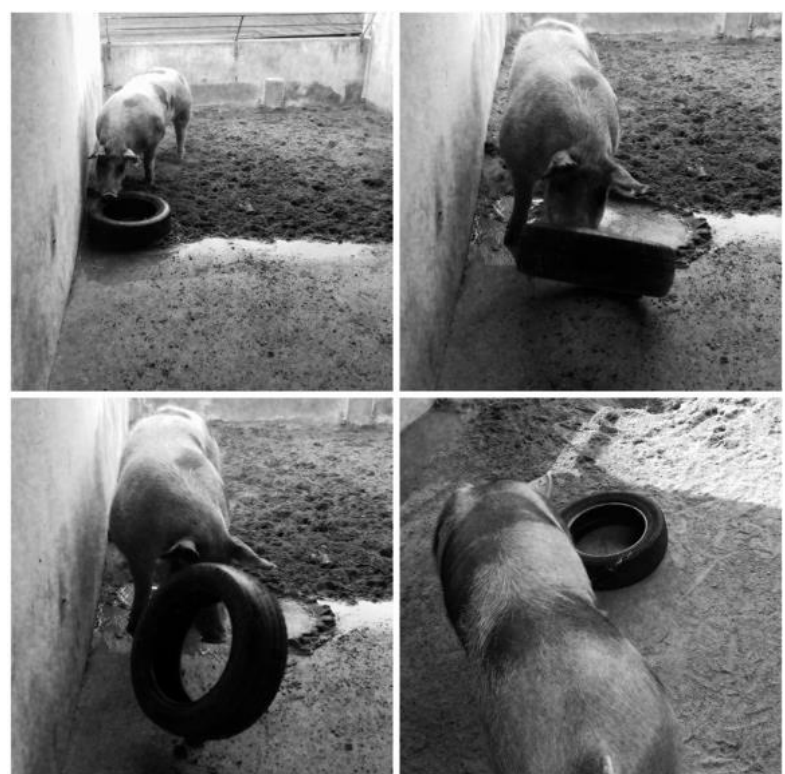

Figura 2. Sequência de interesse e desinteresse de cachaços em relação ao pneu utilizado como enriquecimento ambiental. 
O tempo de interesse dos machos ao enriquecimento foi avaliado a partir do momento em que o animal tinha contato com o objeto até a soltura do mesmo, com observações diretas, sem intervalos, dentro das duas horas. A contagem do tempo foi realizada a partir de um cronometro digital.

Aferiu-se as temperaturas da cabeça, focinho, dorso, ventre e saco escrotal dos animais nos períodos manhã e tarde e das instalações, com mensuração da temperatura do piso de cimento coberto, da área com areia no sol e na sombra, a partir de termômetro de infravermelho (Raytec, modelo Minitemp MT4), em conjunto as análises de interesse.

Tabela 1. Pontuação de interesse e comportamentos avaliados durante a observação de frequência de utilização de enriquecimentos ambientais por cachaços confinados.

\begin{tabular}{ccl}
\hline Objeto & Interesse & \multicolumn{1}{c}{ Comportamento } \\
\hline \multirow{4}{*}{ Corrente } & 0 & Nenhum \\
& 1 & Observar \\
& 1 & Cheirar \\
& 1 & Bater com a cabeça \\
& 1 & Morder \\
& 1 & Empurrar \\
\cline { 2 - 3 } Pneu & 0 & Nenhum \\
& 1 & Observar \\
& 1 & Cheirar \\
& 1 & Morder \\
& 1 & Empurrar com o focinho \\
& 1 & Empurrar com a pata \\
\end{tabular}

A frequência respiratória foi obtida pela contagem dos movimentos do flanco dos animais durante 60 segundos, mensurada no início e no final das observações de interesse.

Realizou-se colheitas de cortisol salivar através de cordas de algodões inseridas individualmente, na baia, na altura da cavidade bucal dos animais, durante quatro dias, de três machos, no período da manhã, em seguida ao término de análise de comportamento de interesse pelo enriquecimento, pelo método de análise quimiolunescência.

A temperatura e a umidade medidas pelos termômetros e dataloguers, instalados próximo ao telhado de madeira, onde foram registradas em intervalos de 15 minutos, durante 24 horas, sempre nos dias das avaliações etológicas e fisiológicas.

Foi realizada análise de tipo de enriquecimento e idade pelo teste do qui-quadrado, correlação de Pearson entre o comportamento e temperatura de superfície corporal e teste-t para frequência respiratória e temperatura do ambiente a partir do software SAS 9.3 (2017).

\section{Resultados e Discussão}

A idade dos machos suínos não influenciou no interesse dos animais pelas correntes e pneus inseridos nas baias $(\mathrm{P}>0,05)$. A idade do animal é um fator importante na escolha do objeto a ser utilizado como enriquecimento ambiental para todas as categorias de suínos. Leitões em fase de maternidade possuem menos interesse com enriquecimentos ambientais que leitões na fase de creche, os quais possuem menos em relação a animais inseridos na fase de crescimento e terminação (Docking et al., 2008).

Neste estudo, $79,86 \%$ dos machos não apresentaram interesse pela corrente, no entanto, $61,11 \%$ dos machos apresentaram interesse pelo pneu. A utilização de correntes e pneus é comum devido sua facilidade de uso e obtenção, no entanto, já está comprovado que os suínos perdem o interesse rapidamente (Guy et al., 2013). O que pode ter acontecido neste estudo, em que o pneu era uma novidade em relação ao material das correntes. Materiais mastigáveis, aromáticos e ingeríveis são mais interessantes para suínos devido à sua curiosidade e alta capacidade investigatória da espécie (Van de Weerd et al., 2003). Segundo Schaefer et al. (1990) pneus suspensos utilizados como enriquecimentos permitiram a apresentação de menos comportamentos agonísticos e melhor taxa de ganho de peso de suínos pós-desmame. Portanto, a escolha do objeto tem que atender aos princípios da espécie, para que os suínos não percam atração por um objeto em curto prazo (Trickett et al., 2009; Campos et al., 2010), atentando-se ainda para a frequência com que os objetivos serão disponibilizados aos animais.

No ambiente controle, $91,67 \%$ dos animais permaneceram em ócio, demonstrando ambiente pobre em entretenimentos. O comportamento apático, definido como a inatividade dos suínos, devido à falta de estimulo ambiental, indica a dificuldade de interação do animal com o meio ambiente (Broom, 1991). Avaliando o comportamento de bem-estar de leitões na fase de creche, a partir da inserção de correntes suspensas, foi observado que os comportamentos de deitar, ficar em pé, chafurdar, comer, fuçar, urinar, brincar e brigar foram mais realizados por animais submetidos ao enriquecimento, mostrando maiores 
atividades dos suínos (Vasconcelos et al., 2015). Tonepohl et al. (2012) verificaram que suínos em crescimento alojados em ambientes com ausência de enriquecimento foram mais agressivos e apresentaram maior nível de cortisol, consequentemente apresentaram lesões graves, prejudicando a qualidade da sua carcaça. Para suínos em fase de crescimento e terminação submetidos a baias com piso semiripado e diferentes áreas de descanso com palha e placa sintética de borracha, estudos indicaram diferenças importantes no comportamento dos animais, com preferência para áreas sujas do que as com placas sintéticas de borracha devido à dificuldade de troca de calor e de manter a homeostasia (Savary et al., 2009).

Não foram encontradas correlações positivas entre temperatura da superfície corporal e comportamento de interesse pelos machos na avaliação do interesse pelos enriquecimentos apresentado pela movimentação física dentro da baia.

As médias de temperatura superficial de dorso, ventre, saco escrotal, focinho e cabeça não diferiram estatisticamente quando avaliadas individualmente em relação aos períodos do dia $(\mathrm{P}>0,05)$.

A frequência respiratória encontrada foi maior no período da tarde, com médias de 28,3 para manhã e 42,3 para tarde $(\mathrm{P}<0,05)$. $\mathrm{O}$ aumento da frequência respiratória permite aumento na perda de calor por evaporação a partir do trato respiratório, que aliado aos demais processos fisiológicos permite a homeostase térmica, em suínos em estresse térmico (Christon, 1998). Em suínos adultos, a frequência respiratória normal pode variar de 15 a 25 movimentos por minuto, indicando estresse térmico frequências acima de 40 movimentos por minuto (Yan e Yamamoto, 2000).

Foram encontradas maiores médias para o período da tarde, com valores de $16^{\circ} \mathrm{C}$ e $22^{\circ} \mathrm{C}$, respectivamente. Em relação aos períodos e as áreas avaliadas, apenas a área de areia na sombra apresentou efeitos significativos $(\mathrm{P}<0,01)$. A temperatura ambiental ideal para os machos suínos, situa-se entre 13 e $21^{\circ} \mathrm{C}$, sendo a crítica inferior igual a $13^{\circ} \mathrm{C}$, e a crítica superior igual a $26^{\circ} \mathrm{C}$ (BRASIL, 1987). Pôde-se observar que os machos, nos períodos mais quentes do dia, procuraram as áreas próximas ao bebedouro, onde havia areia na sombra, e notadamente água, com a formação de poças que permitiam aos animais chafurdar-se. Suínos alojados em ambientes com temperatura alta tendem a permanecem mais próximos ao bebedouro, exibindo maior ingestão de água, menor consumo de alimentos e redução do comportamento de ficar em pé, que influencia negativamente no desempenho dos animais (Kiefer et al., 2009).

Com relação às dosagens séricas de cortisol, houve variações de 0,5 a $3,1 \mathrm{ng} / \mathrm{mL}$ para a semana $1,0,5$ a $0,9 \mathrm{ng} / \mathrm{mL}$ para a semana $2,0,5$ a $1,2 \mathrm{ng} / \mathrm{mL}$ na semana 3 e de 0,9 a $1,2 \mathrm{ng} / \mathrm{mL}$ na semana 4 de avaliação. Na literatura consultada sobre cortisol salivar, foram encontradas médias de 1,2 a 4,5 $\mu \mathrm{g} / \mathrm{dL}$ em avaliações espermáticas e de cortisol de suínos em diferentes períodos do dia, de 74,50 $\mathrm{ng} / \mathrm{mL}$ para animais em pré-abate e $21,70 \mathrm{ng} / \mathrm{mL}$ para suínos alojados em granja comercial. Para suínos em manejo reprodutivo há sugestão de aumento de cortisol, contudo sem indicações de valores de referência (Möstl e Palme, 2002; Santana et al., 2009).

Nesse estudo, os animais não apresentaram dificuldades no momento da colheita de cortisol, mordendo com facilidade a corda de algodão. Barbosa et al. (2013), avaliando a viabilidade de implantação do método de colheita de fluídos a partir de corda de algodão, estimaram o tempo de colheita, volume, qualidade de amostras e comportamento do animal, de suínos mestiços e indicou que a espécie demonstrou interesse pelo método, com competição para mordê-la, com obtenção de amostras em grupo sem estresse aos animais.

Estudos relacionados a melhoria do bemestar animal, incluindo os diferentes tipos de enriquecimento ambiental, são importantes para que alterações significantes e necessárias sejam incorporadas à suinocultura (Maia et al., 2013), com melhorias nos parâmetros econômicos da granja e praticidade para os funcionários, tornando estas alternativas viáveis (Van de Weerd et al. 2009).

Neste estudo o pneu foi inserido no chão das baias e este fato não tornou o objeto menos atrativo, demonstrando que são várias as formas de se utilizar um mesmo objeto, podendo de maneira correta reaproveita-lo. As correntes são amplamente utilizadas, e para suínos machos elas apresentam grande utilidade como enriquecimento ambiental.

\section{Conclusão}

O enriquecimento ambiental é uma alternativa viável para todas as fases de suínos em 
sistema de confinamento. Quando utilizado em instalações para machos suínos reprodutores constitui-se como uma importante ferramenta para melhorar o bem-estar dos animais, sendo o pneu o melhor método de entretenimento quando comparado ao uso de correntes.

\section{Conflito de Interesse}

Os autores declaram não existir conflito de interesse.

\section{Comitê de Ética}

O projeto foi aprovado pela Comissão de Ética no Uso de Animais (CEUA) da Faculdade de Zootecnia e Engenharia de Alimentos da Universidade de São Paulo - FZEA/USP, sob o protocolo CEUA $\mathrm{n}^{\mathrm{o}}$ 3758260116, sob a responsabilidade de Cristiane Gonçalves Titto.

\section{Referências}

Baptista, R.I.A.A.; Bertani, G.R.; Barbosa, C.N. Indicadores do bem-estar em suíno. Ciência Rural, 41(10): 1823-1830, 2011.

Barbosa, C.N.; Baptista R.I.A.A.; Cunha, D.F.; Freitas, T.R.P. Aplicação da metodologia de coleta do fluido oral em suínos mestiços. Medicina Veterinária, 7(3): 32-38, 2013.

Bloomsmith, M.A.; Brent, L.Y.; Schapiro, S.J. Guidelines for developing and managing an environmental enrichment program for nonhuman primates. Laboratory Animal Science, 4(41): 372-377, 1991.

BRASIL. Empresa Brasileira de Pesquisa Agropecuária. Efeito de diferentes sistemas de aquecimento no desempenho de leitões, de $1987 . \quad$ Disponível em: <https://www.embrapa.br/busca-depublicacoes/-/publicacao/435571/efeito-dediferentes-sistemas-de-aquecimento-nodesempenho-de-leitoes>. Acesso em: 30 out. 2017

BRASIL. Empresa Brasileira de Pesquisa Agropecuária. Efeito do tempo de jejum dos suínos na granja sobre o bem-estar, medido pelo cortisol na saliva e pela frequência cardíaca, durante o manejo pré-abate, de $2006 . \quad$ Disponível em: <https://www.infoteca.cnptia.embrapa.br/bitstr eam/doc/443744/1/publicacaop9p49c0o.pdf>. Acesso em: 30 out. 2017

Broom, D.M. Animal welfare: concepts and measurements. Journal of Animal Science, 69(10): 4167-4175, 1991.
Broom, D.M.; Molento, C.F.M. Bem-estar animal: conceitos e questões relacionadas - Revisão. Archives of Veterinary Science, 9(2): 1-11, 2004.

Campos, J.A.; Tinôco, I.F.F.; Silva, F.F.; Pupa, J.M.R.; Silva, I.J.O. Enriquecimento ambiental para leitões na fase de creche advindos de desmame aos 21 e 28 dias. Revista Brasileira de Ciências Agrárias, 5(2): 272-278, 2010.

Christon R. The effect of tropical ambient temperature on growth and metabolism in pigs. Journal Animal Science, 66(5): 31123123, 1998.

Docking, C.M.; van de Weerd, H.A.; Day, J.E.L.; Edwards, S.A. The influence of age on the use of potential enrichment objects and synchronisation of behaviour of pigs. Applied Animal Behaviour Science, 110(2): 244-257, 2008.

Ferreira, R.A.; Fialho, E.T.; Lima, J.A.F. Criação técnica de suínos. Lavras: Universidade Federal de Lavras, 2004. 59p.

Foppa, L.; Caldara, F.R.; Machado, S.P.; Moura, R.; Santos, R.K.S.; Nããs, I.A.; Garcia, R.G. Enriquecimento ambiental e comportamento de suínos: revisão. Brazilian Journal of Biosystems Engineering, 8(2): 7-15, 2014.

Fraser, A.F.; Broom, D.M. Farm animal behaviour and welfare. Reino Unido: Ballière Tindall, 1990. 437p.

Guy, J.H., Rowlinson, P., Chadwick, J.P., Ellis, M. Behaviour of two genotypes of growingfinishing pigs in three different housing systems. Applied Animal Behavior Science. 75(1): 193-206, 2002.

Guy, J.H.; Meads, Z.A.; Shiel, R.S.; Edwards, S.A. The effect of combining different environmental enrichment materials on enrichment use by growing pigs. Applied Animal Behaviour Science, 144(23): 102-107, 2013.

Maia A.P.A.; Sarubbi, J.; Medeiros, B.B.L.; Moura, D.J. Enriquecimento ambiental como medida para o bem-estar positivo de suínos. Revista do Centro de Ciências Naturais e Exatas, 14(5): 2862-2877, 2013.

Manteca, X.; Silva, C.A.; Bridi, A.M.; Dias, C.P. Bem-Estar Animal: Conceitos e Formas Práticas de Avaliação dos Sistemas de Produção de Suínos. Ciências Agrárias, 34(6): 37-46, 2013.

Mench J.A. Environmental enrichment and the importance of exploratory behavior. In: 
Shepherdson, D.J.; Mellen, J.D.; Hutchins, M. Second nature: environmental enrichment for captive animals. Washington: Smithsonian Institution Press, 1998. p.30-46.

Möstl, E.; Palme, R. Hormones as indicators of stress. Domestic animal endocrinology, 23: 67-74, 2002.

Newberry R.C. Environmental enrichment: increasing the biological relevance of captive environments. Applied Animal Behaviour Science, 44(3): 229-243, 1995.

Oliveira, A.L.S.; Donzele, J.L.; Oliveira, R.F.M.; Ferreira, A.S.; Generoso, R.A.R. Lisina em rações para suínos machos castrados selecionados para deposição de carne magra na carcaça dos 110 aos $125 \mathrm{~kg}$. Revista Brasileira de Zootecnia, 32(1) :150-155, 2003.

Pereira, M. L. Biodigestores: opção tecnológica para a redução dos impactos ambientais na suinocultura. Disponível em: <http://www.embrapa.br>. Acesso em: 20 out. 2017

Rodarte, L.F. The effect of environmental manipulation on behavior, salivary cortisol and growth of piglets weaned at 14 days of age. Journal of Applied Animal Welfare Science, 7: 171-179, 2004.

Santana, A.P.; Murata, L.S.; McManus, C.P.; Bernal, F.E.M. Dosagem de cortisol sanguíneo em suínos submetidos ao manejo pré-abate e insensibilização elétrica. Archivos Zootecnia, 58(221): 149-152, 2009.

Sarubbi, J.; Rossi, L.A.; Moura, D.J.; Oliveira, R.A.; David, E. Utilização de energia elétrica em diferentes sistemas de aquecimento para leitões. Engenharia Agrícola, 30(6): 10031011, 2010.

Savary, P.; Gygax, L.; Wechsler, B.; Hauser, R. Effect of a synthetic plate in the lying area on lying, behaviour, degree of fouling and skin lesions at the leg joints of finishing pigs. Applied Animal Behavior Science, 118(1): 20-27, 2009.
Schaefer, A.L.; Salomons, M.O.; Tong, A.K.W.; Sather, A.P.; Lepage, P. The effect of environment enrichment on aggression in newly weaned pigs. Applied Animal Behaviour Science, 27(2): 41-52, 1990.

Scott, K.; Taylor, L.; Gill, B.P.; Edwards, S.A. Influence of different types of environmental enrichment on the behaviour of finishing pigs in two different housing systems: 3 . Hanging toy versus rootable toy of the same material. Applied Animal Behaviour Science, 116(2): 186-190, 2009.

Trickett, S.L.; Guy, J.H.; Edwards, S.A. The role of novelty in environmental enrichment for the weaned pig. Applied Animal Behaviour Science, 16(1): 45-51, 2009.

Van De Weerd, H. A., Docking, C. M., Day, J. E. L., Avery, P. J., Edwards, S. A. A systematic approach towards developing environmental enrichment for 15 pigs. Applied Animal Behavior Science, 84(2), 101-118. 2003.

Van de Weerd, H.A.; Docking, C.M.; Day, J.E.L.; Breuer, K.; Edwards, S.A. Effects of species relevant environmental enrichment on the behaviour and productivity of finishing pigs. Applied Animal Behaviour Science, 99(1): 230-247, 2006.

Van de Weerd, H.A.; Day, J.E.L. A review of environmental enrichment for pigs housed in intensive housing systems. Applied Animal Behaviour Science, 116(1): 1-20, 2009.

Vasconcelos, E.K.F.; Borges, L.S.; Silva, A.L.; Andrade, T.V.; Santos, E.T.; Sousa Jr, S.C.; Farias, L.A. Comportamento de suínos na fase de crescimento criados em ambiente enriquecido. Journal of Animal Behaviour and Biometeorology, 3(2): 120-123, 2015.

Yan, P. S.; Yamamoto, S. Relationship between thermoregulatory responses and heat loss in piglets. Journal of Animal Science, 71(10): 5005-509, 2000. 